\title{
MOTIVAÇÃO, BEM-ESTAR E AUTOESTIMA DE PRATICANTES DE DIFERENTES MODALIDADES DE EXERCÍCIO FÍSICO
}

\section{Thays da Cruz Silva}

Mestranda em Psicologia pela Universidade Federal do Vale do São Francisco - Univasf, Petrolina/PE, Brasil

\section{Gabriel Lucas Morais Freire}

Mestrando em Educação Física pela Universidade Federal do Vale do São Francisco - Univasf, Petrolina/ PE, Brasil.

\section{Olga Santana Guimarães Morais}

Mestranda em Psicologia pela Universidade Federal do Vale do São Francisco - Univasf, Petrolina/PE, Brasil.

José Roberto A. do Nascimento Junior

Doutorado em Educação Física pelo Programa de Pós-graduação Associado UEM/UEL. Doutorado Sanduíche como pesquisador visitante em Estudos do Esporte, na linha de Psicologia do Esporte pela University of Stirling, Escócia, Reino Unido.
RESUMO: Este estudo transversal investigou a motivação, o bem-estar e a autoestima entre 31 praticantes (24 mulheres e 7 homens) com média de idade de 30,41 anos $( \pm 7,85)$ de três modalidades de exercício (treinamento funcional $(n=10)$, musculação $\operatorname{com}(n=11)$ e sem $(\mathrm{n}=10)$ personal trainer $)$ de academias das cidades de Petrolina (PE) e Juazeiro (BA). Foram utilizados a Escala de Bem-Estar Subjetivo, a Escala de Autoestima de Rosenberg e o Questionário de Regulação de Conduta no Exercício 2. Os resultados evidenciaram que os praticantes de treinamento funcional apresentaram maior afeto positivo e menor escore de regulação identificada $(r=-0,34)$ do que os praticantes de musculação com e sem personal. Verificaram-se as seguintes correlações significativas: afeto positivo com regulação identificada ( $\mathrm{r}$ $=-0,34)$, regulação introjetada $(\mathrm{r}=-0,31)$ e regulação externa $(\mathrm{r}=$ $-0,30)$; e satisfação com a vida com regulação identificada $(r=-0,28)$. Pode-se concluir que o tipo de exercício pode ser considerado um fator interveniente no afeto positivo e na identificação do indivíduo com o exercício, e que o afeto positivo está inversamente associado às regulações da motivação.

PALAVRAS-CHAVE: Motivação; Bem-estar; Autoestima; Exercício.

\section{MOTIVATION, WELL-BEING AND SELF-ESTEEM OF PEOPLE PRACTICING DIFFERENT PHYSICAL EXERCISES}

\begin{abstract}
Current transversal study investigates motivation, wellbeing and self-esteem between 31 people ( 24 females and 7 males), mean age 30.41 years $( \pm 7.85)$, practising three modalities (functional training $(\mathrm{n}=10)$, bodybuilding $(\mathrm{n}=11)$ and without personal trainer $(\mathrm{n}=10))$ in gyms in Petrolina and Juazeiro, Brazil. Subjective WellBeing Scale, Rosemberg's Self-Esteen Scale and the Questionnaire of Conduct regulation in Exercise 2 were assessed. Practitioners of functional training had the highest positive rate and the least score in identified regulation $(r=-0.34)$ when compared to bodybuilding with or without personal trainers. Significant co-relationships occurred: positive affection with identified regulation $(\mathrm{r}=-0.34)$, introjected regulation $(\mathrm{r}=-0.31)$ and external regulation $(\mathrm{r}=-0.30)$; and satisfaction with life with identified regulation $(r=-0.28)$. The type of exercise may be considered an intervening factor in positive affection and in the individual's identification with the exercise. Moreover, positive affection is inversely associated with motivation regulations.
\end{abstract}

KEY WORDS: Motivation; Well-being; Self-esteem; Exercise.
Gabriel Morais

bi88el@gmail.com 


\section{INTRODUÇão}

Apesar de na literatura ser consenso que a prática regular de exercício físico está associada à melhora dos aspectos físicos, fisiológicos e psicológicos ${ }^{1-3}$, apenas aproximadamente $40 \%$ da população brasileira pratica algum esporte ou exercício físico ${ }^{4}$. Nessa perspectiva, a Organização Mundial de Saúde (OMS) ${ }^{5}$ dá alertas para a necessidade do engajamento da população em programas de atividade física com o intuito de reduzir o sedentarismo e as consequências negativas que este pode provocar para a saúde física e mental.

A literatura aponta que a adesão e permanência na prática de exercício físico está diretamente associada à motivação do indivíduo pela modalidade ${ }^{6,7}$. A motivação é caracterizada como uma ação intencional, direcionada para uma meta e regulada por fatores ambientais e pessoais ${ }^{8}$. Uma das principais abordagens para o estudo da motivação tem sido a Teoria da Autodeterminação (TAD) ${ }^{8,9}$, que tem o propósito de interpretar a personalidade da motivação humana, e foi desenvolvida para responder por que uma parcela da população é mais intrinsecamente motivada do que outras, apresentando comportamentos mais enérgicos, vitais e em busca de aprendizado e bem-estar, enquanto que outra parte demonstra atitudes contrárias como apatia e falta de interesse em suas atividades.

A TAD indica que a motivação se desenvolve por meio de um continuum, derivada de seis regulações de motivação $0^{8}$, que vai do prazer em realizar algo até a falta de interesse ou ausência de motivação, que é a desmotivação. A regulação externa é a forma da motivação extrínseca, na qual o indivíduo é regulado por fatores externos, como a recompensa, reconhecimento social, ou para evitar punição ${ }^{8}$. A regulação introjetada é o comportamento controlado por pressões internas causadas por influências extrínsecas ${ }^{8,9}$. Já a regulação identificada ocorre quando existe percepção de que o comportamento é relevante para a vida. Por fim, a regulação intrínseca é considerada o estilo de autodeterminação perfeito, no qual as atividades são realizadas simplesmente pela satisfação ao se realizar. Estudos recentes têm aplicado a TAD em distintos grupos populacionais (idosos ${ }^{10-12}$ e adultos ${ }^{13,14}$ ) e em diferentes contextos do exercício (musculação $0^{15,16}$, crossfit $\left.{ }^{13,14}\right)$.
A TAD ressalta que a motivação do indivíduo para se engajar em determinado exercício está associada à percepção que o indivíduo tem de si mesmo (autoestima) e sentimentos relativos a si mesmo e às atividades que realiza (bem-estar) ${ }^{17}$, uma vez que o engajamento em programas de exercício físico tem como um dos eixos centrais a socialização como forma de motivação e autoestima para a prática ${ }^{18}$. Bavoso et al. ${ }^{11}$ observaram associação entre a autoestima e a motivação para o exercício físico em adultos e idosos praticantes de academia, com intuito de conhecer sobre o que motiva os participantes pela procura do exercício físico. Especificamente, os autores verificaram que a autoestima dos idosos está associada a motivos como manutenção de saúde e convívio social, enquanto nos adultos a autoestima tem relação multifatorial com os motivos para a prática do exercício. Rosário et al. ${ }^{12}$ observaram que a motivação intrínseca para a prática do exercício favorece o desenvolvimento do bem-estar subjetivo dos praticantes, além de levar ao aumento do nível da vitalidade, satisfação com a vida e autoestima.

Dentre os diversos tipos de exercício existentes, a musculação e o treinamento funcional são modalidades com grande procura e adesão nos últimos anos ${ }^{19,20}$, e que favorecem a melhora da autoestima e do bem-estar subjetivo ${ }^{14}$. A musculação é definida como um treinamento de força, que é considerado o método mais eficaz para aumentar a força e massa muscular ${ }^{21,22}$. Nos últimos anos a utilização dos serviços do personal trainer para a prática da musculação tem aumentado nas academias, uma vez que a prescrição do exercício é elaborada exclusivamente para o indivíduo, com foco nos seus objetivos, bem-estar, motivação e autoestima ${ }^{23}$, sendo o contexto social um fator importante para o engajamento ativo e crescimento psicológico dos praticantes ${ }^{8,9}$. Já o treinamento funcional tem como finalidade preparar o corpo de uma forma integral por meio de um centro corporal, abarcando o grupo muscular dos transversos espinhais, diferente da musculação o treinamento funcional as atividades são elaboradas ) rever redação!! para treinos em grupo, com foco nos objetivos do grupo, bem-estar e motivação ${ }^{24}$.

Embora a adesão de indivíduos na prática dessas modalidades, ainda são escassas as pesquisas que comparam os atributos psicológicos de praticantes de diferentes modalidades de exercício ${ }^{21-2}$, principalmente, entre os praticantes de treinamento personalizado e treinamento 
funcional. Nessa perspectiva, este estudo se torna relevante na medida em que poderá proporcionar informações para os profissionais que trabalham com prescrição de exercício a respeito dos atributos psicológicos inerentes a cada modalidade e o quanto o tipo de exercício pode interferir nestes atributos. Diante disso, o presente estudo teve como objetivo investigar a motivação, o bem-estar e a autoestima entre praticantes de diferentes modalidades de exercício físico, além de verificar as relações entre as variáveis. Como hipótese, espera-se que os indivíduos que se utilizam do serviço de personal trainer apresentarão maior percepção de motivação, autoestima e bem-estar do que os indivíduos que não possuem esse serviço e os praticantes de treinamento funcional.

\section{MATERIAIS E MÉTODOS}

\section{PARTICIPANTES}

Participaram desse estudo 31 indivíduos de ambos os sexos (24 mulheres e 7 homens) com média de idade de 30,41 \pm 7,85 anos, de três modalidades de exercício físico de uma academia com sede na cidade de Petrolina (PE) e filial na cidade de Juazeiro (BA): musculação $(n=10)$, musculação com personal trainer $(n=11)$ e treino funcional $(n=10)$. A amostra foi selecionada de forma não probabilística e por conveniência. A média de frequência semanal de prática de exercício foi de 4,0 \pm 7,85 vezes por semana. Todos os participantes assinaram voluntariamente o Termo de Consentimento Livre e Esclarecido. Os critérios estabelecidos para a inclusão dos participantes no estudo foram a idade mínima de 18 anos e ser praticante de uma das modalidades já citadas há, no mínimo, três meses.

\section{INSTRUMENTOS}

A Escala de Bem-Estar Subjetivo (EBES) avalia três componentes do bem-estar subjetivo: afeto positivo, afeto negativo e satisfação com a vida. Os 62 itens são respondidos a partir de pontos que variam de 1 (nem um pouco) a 5 (extremamente). Esta escala foi elaborada inicialmente por Lawrence et al. ${ }^{25} \mathrm{e}$ validada para o contexto brasileiro por Albuquerque et al. ${ }^{26}$, e na versão validada possui duas dimensões: na primeira, correspondente aos afetos positivos e negativos, o indivíduo deve responder como tem se sentido ultimamente; já na segunda, devem ser feitos julgamentos a respeito da satisfação com a vida.

A Escala de Autoestima de Rosenberg avalia a autoestima global por meio de dez itens de sentimentos de autoestima e autoaceitação que são respondidos em uma escala likert de 5 pontos num continuum de (1) discordo totalmente (1) a (5) concordo totalmente. Pontuações inferiores a 15 demonstram um nível de autoestima baixo. Ela possui o nome de seu criador e sua validação para o português foi revisada por Hutz et al. ${ }^{27}$.

O Questionário de Regulação de Conduta no Exercício (BREQ-2) foi utilizado para verificar o nível de motivação para a prática do exercício ${ }^{28}$. O BREQ2 é baseado na Teoria da Autodeterminação e procura quantificar as regulações motivacionais (internas e externas), além de quantificar a amotivação relacionada à prática de exercícios. O instrumento é composto por 19 itens com cinco opções de resposta, em uma escala likert, em um continuum de "não é verdade pra mim" (0) a "muitas vezes é verdade pra mim" (4). Os itens são distribuídos em cinco dimensões: amotivação, regulação externa, regulação introjetada, regulação identificada e motivação intrínseca.

\section{PROCEDIMENTOS}

A pesquisa é integrante do projeto institucional aprovado sob parecer no 2.442 .590 do Comitê de Ética em Pesquisa com Seres Humanos da Univasf, Petrolina-PE. Inicialmente foi solicitada autorização das academias para a realização das coletas de dados com os alunos. Os participantes foram convidados a participar da pesquisa por meio da assinatura do Termo de Consentimento Livre e Esclarecido. A coleta de dados ocorreu no primeiro semestre de 2018. As coletas foram realizadas nas próprias academias. A aplicação dos questionários foi realizada de forma individual, em uma sala privativa e o preenchimento dos questionários teve duração de aproximadamente 20 minutos. A ordem dos questionários foi aleatorizada entre os participantes. 


\section{ANÁLISE DOS DADOS}

Para a análise dos dados, foi utilizada frequência e percentual para as variáveis categóricas. Para as variáveis numéricas, inicialmente foi verificada a normalidade dos dados por meio do teste Shapiro-wilk. Como os dados não apresentaram distribuição normal, as medidas de tendência central e dispersão utilizadas para descrever os resultados foram a mediana (Md) e o intervalo interquatílico (Q1; Q3) para a caracterização dos resultados. $\mathrm{Na}$ comparação entre as modalidades foi utilizado o teste de Kruskal-wallis seguido do teste "U" de Mann-whitney para pares de grupos. A correlação entre as variáveis foi efetuada por meio do coeficiente de correlação de Spearman. A significância adotada foi de $\mathrm{p}<0,05$. Os dados foram analisados no software SPSS 22.0.

\section{RESULTADOS}

Ao comparar a motivação, o bem-estar e a autoestima entre os praticantes de treinamento funcional, musculação com personal e musculação sem personal (Tabela 1), em relação à motivação, foi encontrada diferença significativa apenas na regulação identificada $(\mathrm{p}$ $=0,006$ ), indicando que os praticantes de treinamento funcional apresentaram menor nível de motivação nesta regulação do que os praticantes de musculação com e sem personal. No que se refere ao bem-estar subjetivo (Tabela 1), houve diferença significativa entre os grupos no afeto positivo do bem-estar subjetivo ( $p=0,017$ ), evidenciando que os praticantes de treinamento funcional apresentaram maior escore do que os praticantes de musculação com e sem treinamento personalizado. Em relação à autoestima, não houve diferença significativa $(\mathrm{p}$ $>0,05$ ) no nível de autoestima entre os grupos (Tabela 1), indicando que os praticantes das três modalidades apresentaram escore moderado de autoestima.

Conforme os resultados expressos na Tabela 2, verificaram-se as seguintes correlações significativas ( $\mathrm{p}<$ $0,05)$, negativas e fracas $(\mathrm{r}<0,40)$ entre as variáveis: afeto positivo e regulação identificada $(r=-0,34)$, afeto positivo e regulação introjetada $(r=-0,31)$ e afeto positivo com regulação externa $(r=-0,30)$, satisfação com a vida e regulação identificada $(r=-0,28)$. Estes resultados evidenciam uma associação inversamente proporcional do afeto positivo (bem-estar) com as regulações da motivação extrínseca, demonstrando que o afeto negativo pode ser prejudicado pela motivação extrínseca. Além disso, a satisfação com a vida também se associou de forma inversamente proporcional com a regulação identificada, indicando que a identificação com o exercício está associada a pior percepção de satisfação com a vida.

\section{DISCUSSÃO}

A presente investigação objetivou investigar a motivação, bem-estar e autoestima de praticantes diferentes modalidades de exercício físico. Os principais achados foram que os praticantes de treinamento funcional apresentaram maior afeto positivo (bem-estar) do que os praticantes de musculação com e sem personal trainer, enquanto que os praticantes de treinamento funcional apresentaram maior identificação (motivação) com a modalidade (Tabela 1). Além disso, verificou-se associação negativa do afeto positivo com as regulações menos autodeterminadas de motivação (Tabela 2).

O principal resultado deste estudo se refere a maior percepção de felicidade, prazer (afeto positivo) entre os praticantes de treinamento funcional em detrimento aos praticantes de musculação com e sem treinamento personalizado (Tabela 1), contradizendo a hipótese do estudo. Indicando que o treinamento funcional pode proporcionar mais sentimento momentâneo de prazer e entusiasmo durante a prática do exercício ${ }^{29}$. Este resultado pode estar relacionado ao fato do treinamento funcional ser realizado em grupos e com exercícios mais dinâmicos em comparação à musculação, que engloba exercícios mais estáticos e realizados individualmente. Almeida, Pereira e Fernandes ${ }^{29}$ afirmam que a prática de atividades coletivas favorece de forma mais significativa a promoção do bem-estar subjetivo. De acordo com a TAD, o contexto social é determinante para o desenvolvimento do comportamento autodeterminado e para que o indivíduo sinta prazer e satisfação pela tarefa a ser executada ${ }^{8}$, o que também pode explicar o maior afeto positivo entre os praticantes de treinamento funcional. Esses achados corroboram com os de Fisher et al. ${ }^{13}$, que encontraram resultados semelhantes comparando praticantes de crossfit e indivíduos que praticam musculação, com ou sem personal trainer. Embora, a autoestima não apresentou diferença com nenhuma das outras variáveis, indicando que qualquer uma das modalidades apresentando escores moderados relacionado à autoestima. 
Tabela 1. Comparação da motivação para o exercício, bem-estar subjetivo e autoestima entre os praticantes de treinamento funcional, musculação com personal trainer e musculação sem personal trainer das cidades de Juazeiro-BA e Petrolina-PE

\begin{tabular}{|c|c|c|c|c|}
\hline \multirow[t]{2}{*}{ VARIÁVEIS } & $\begin{array}{c}\text { 1. Treinamento } \\
\text { funcional }(n=10)\end{array}$ & $\begin{array}{l}\text { 2. Musculação com } \\
\text { personal }(\mathrm{n}=11)\end{array}$ & $\begin{array}{l}\text { 3. Musculação sem } \\
\text { personal }(\mathrm{n}=10)\end{array}$ & \multirow[t]{2}{*}{ p } \\
\hline & Md (Q1-Q3) & Md (Q1-Q3) & Md (Q1-Q3) & \\
\hline \multicolumn{5}{|l|}{ Motivação } \\
\hline Regulação intrínseca & $3,50(2,75-4,00)$ & $3,50(2,75-4,00)$ & $3,50(2,75-3,62)$ & 0,943 \\
\hline Regulação identificada & $2,75(2,00-3,31)^{b}$ & $3,75(3,25-4,00)$ & $3,25(3,00-3,75)$ & $0,006 *$ \\
\hline Regulação introjetada & $1,00(0,50-2,00)$ & $2,67(1,67-3,33)$ & $1,83(0,91-2,41)$ & 0,146 \\
\hline Regulação externa & $00(00-0,06)$ & $0,5(00-1,0)$ & $00(00-1,12)$ & 0,097 \\
\hline Desmotivação & $00(00-0,12)$ & $00(00-00)$ & $00(00-00)$ & 0,346 \\
\hline \multicolumn{5}{|l|}{ Bem-estar } \\
\hline Afeto positivo & $3,54(3,31-3,72)^{\mathrm{a}}$ & $3,14(2,90-3,29)$ & $3,09(2,86-3,37)$ & $0,017^{*}$ \\
\hline Afeto negativo & $2,29(2,03-2,45)$ & $2,27(1,92-2,54)$ & $2,26(2,10-2,63)$ & 0,724 \\
\hline Satisfação com a vida & $3,00(2,72-3,15)$ & $3,00(2,50-3,13)$ & $2,93(2,59-3,25)$ & 0,824 \\
\hline Autoestima & $25,50(24,25-27,25)$ & $25,00(22,00-27,00)$ & $25,00(23,00-28,00)$ & 0,884 \\
\hline
\end{tabular}

*Diferença significativa - $\mathrm{p}<0,05$ : Teste de Kruskal-Wallis entre: a, b) 1 com 2 e 3. Nota: Md = Mediana; Q1 = Quartil 1; Q3 $=$ Quartil 3 .

Tabela 2. Correlação entre a motivação para o exercício, o bem-estar subjetivo, e a autoestima dos praticantes de musculação e treinamento funcional

\begin{tabular}{|c|c|c|c|c|c|c|c|c|c|}
\hline \multirow{2}{*}{ VARIÁVEIS } & \multicolumn{5}{|c|}{ Motivação } & \multicolumn{3}{|c|}{ Bem-estar } & \multirow{2}{*}{$\begin{array}{c}\text { Autoestima } \\
9\end{array}$} \\
\hline & 1 & 2 & 3 & 4 & 5 & 6 & 7 & 8 & \\
\hline \multicolumn{10}{|l|}{ Motivação } \\
\hline 1. Regulação intrínseca & & $-0,01$ & 0,25 & $-0,21$ & $-0,13$ & $-0,09$ & $-0,18$ & $-0,08$ & $-0,19$ \\
\hline 2. Regulação identificada & & & 0,23 & 0,15 & $-0,10$ & $-0,34^{*}$ & $-0,01$ & $-0,28^{*}$ & 0,08 \\
\hline 3. Regulação introjetada & & & & $0,48^{*}$ & 0,22 & $-0,31^{*}$ & $-0,13$ & 0,07 & 0,05 \\
\hline 4. Regulação externa & & & & & 0,19 & $-0,30^{*}$ & 0,08 & 0,06 & 0,14 \\
\hline 5. Amotivação & & & & & & $-0,16$ & $-0,17$ & 0,14 & 0,29 \\
\hline \multicolumn{10}{|l|}{ Bem-estar } \\
\hline 6. Afeto positivo & & & & & & & 0,11 & 0,12 & 0,16 \\
\hline 7. Afeto negativo & & & & & & & & 0,25 & 0,08 \\
\hline 8. Satisfação com a vida & & & & & & & & & 0,21 \\
\hline 9. Autoestima & & & & & & & & & \\
\hline
\end{tabular}

*Correlação significativa $(\mathrm{p}<0,05)$ - Correlação de Spearman. 
Em contrapartida, os praticantes de treinamento funcional demonstraram menor regulação identificada em detrimento aos praticantes das outras modalidades (Tabela 1), indicando que os praticantes de musculação com e sem personal trainer se identificam mais com o exercício e o percebem como importante para as suas vidas. Como a musculação é uma modalidade mais popular que apresenta maior identificação e seus objetivos mais evidenciados na literatura de serem alcançados como, por exemplo, diminuição da gordura visceral e triglicerídeos, aumento da massa muscular e massa óssea ${ }^{30}$. Caudwell e Keatley $^{31}$ argumentam que praticantes de musculação podem ser mais motivados, sem a contingências externas, tais como recompensas ou pressão social.

Segundo a $\mathrm{TAD}$, este tipo de interesse ocorre pelo fato da adesão ao exercício de musculação ser maior quando os motivos intrínsecos estão relacionados ao prazer e sentimento de competência, em comparação com os resultados relacionados com o corpo ${ }^{8,9}$. Esses achados vão de encontro com os de Marin et al. ${ }^{14}$ que compararam o nível de motivação entre praticantes de crossfit e treinamento de força, demostrando que os praticantes de crossfit relataram níveis elevados de motivação intrínseca para o exercício, enquanto os praticantes de treinamento de força apresentaram maior motivação para regulação externa. Fisher et al..$^{13}$ também observaram que os praticantes de crossfit relataram níveis mais elevados de motivação intrínseca, como prazer e desafio, enquanto os praticantes de musculação que treinavam com personal trainer relataram valores mais elevados por motivos de saúde. Os autores afirmam ainda que no crossfit as motivações são semelhantes aos observados na participação em esportes, podendo ter influência sobre a adesão dos participantes em comparação aos participantes do treinamento resistido. A partir dos princípios da TAD pode-se argumentar que o processo de desenvolvimento da motivação está relacionado tanto a modalidade quanto a maneira que os participantes percebem suas competências com relacionamentos sociais durante a prática ${ }^{8,9}$.

Esses resultados também podem estar relacionados ao fato de uma ter maior cobrança dos professores e instrutores de treinamento funcional durante as aulas, o que pode desmotivar o praticante quando o mesmo não consegue realizar os exercícios com competência e autonomia ${ }^{31}$. Teixeira et al. ${ }^{32}$ observaram que o prazer proporcionado pelo exercício pode proporcionar maior persistência, diminuição do estresse, confiança e satisfação com a prática. Dessa maneira, os profissionais envolvidos nesta modalidade devem sempre buscar proporcionar esse tipo de situação aos seus praticantes. De acordo com a TAD, os indivíduos são intrinsecamente motivados quando se envolvem em uma atividade para a satisfação inerente que eles derivam da atividade, sendo uma motivação mais autodeterminada ${ }^{8,9}$.

Pode-se perceber que as regulações menos autodeterminadas da motivação apresentaram associação inversamente proporcional com o afeto positivo (Tabela 2), evidenciando que quanto mais o comportamento do praticante é regulado para a prática do exercício por fatores externos, menor o sentimento momentâneo de prazer proporcionado pela atividade ${ }^{33}$. Esse achado demonstra que quando o afeto pela atividade é alto, menores são as chances de indivíduos realizarem o exercício como uma obrigação e sim como forma de prazer. Balbinotii et al. ${ }^{34}$ observaram que a satisfação em realizar o exercício/atividade apresentou capacidade preditiva sobre a motivação. Com isso, o aumento do afeto positivo reflete na extensão que o indivíduo se sente entusiasmado, ativo e alerta para o exercício tornando improvável que a prática seja algo desmotivante ${ }^{8-10}$.

Além disso, verificou-se que quanto mais o praticante de exercício está satisfeito com a vida (motivação, bem-estar e autoestima), menos se identifica com a modalidade praticada (Tabela 2). Este achado demonstra que quando os indivíduos estão satisfeitos com suas condições físicas, psicológicas e sociais, menos eles demostram interesse na prática do exercício ${ }^{32,35}$.

Apesar das contribuições para a literatura a respeito da motivação, da autoestima e do bem-estar em praticantes de diferentes modalidades de exercício físico, algumas limitações precisam ser apontadas. Primeiramente, o tamanho reduzido da amostra, a não equivalência entre os sexos e a restrição geográfica não permitem a generalização dos resultados. Outra limitação se refere ao desenho transversal do estudo, impossibilitando inferências de causalidade entre as variáveis. Dessa forma, futuras investigações devem continuar a explorar as relações entre as variáveis a partir de um desenho longitudinal, 
com amostras mais representativas, com praticantes das mesmas modalidades e em praticantes de outras modalidades, como o crossfit e as artes marciais.

\section{CONCLUSÃO}

Pode-se observar que houve diferença entre a motivação e o bem-estar em praticantes de diferentes tipos de exercício, sendo a autoestima a única a não apresentar diferença. A partir dos resultados obtidos, pode-se concluir que o tipo de exercício pode ser considerado um fator interveniente na motivação (identificada) e no bem-estar (afeto positivo) com o exercício. Ressalta-se que a prática de exercícios em grupo, como o treinamento funcional, pode favorecer o desenvolvimento do bem-estar subjetivo, entretanto, este tipo de exercício parece não levar a motivação (estilo regulatório de identificação) e valorização da atividade pelo indivíduo. Além disso, percebeu-se uma relação inversamente proporcional entre as regulações da motivação extrínseca e o bem-estar subjetivo. Do ponto de vista prático, os achados da presente investigação contribuem para auxiliar na compreensão de como as diferentes modalidades de exercício físico podem interferir nos atributos psicológicos do praticante, especificamente na motivação e no bem-estar subjetivo. Com isso, esses resultados podem auxiliar os profissionais do exercício físico na forma de intervenção com esta população, bem como prescrição do treinamento e da retenção dos mesmos em suas modalidades.

\section{REFERÊNCIAS}

1. Agostini CM, Rodrigues VS, Guimarães AC, Damázio LCM, Vasconcelos NN. Análise do desempenho motor e do equilíbrio corporal de idosos ativos com hipertensão arterial e diabetes tipo 2. Rev Aten Saú (antiga Rev Bras Ciên Saúde). 2018;16(55):29-35.

2. Chung P-K, Zhao Y, Liu J-D, Quach B. A canonical correlation analysis on the relationship between functional fitness and health-related quality of life in older adults. Arch Gerontol Geriatr. 2017;68:44-8.

3. Siqueira AF, Rebesco DB, Amaral FA, Maganhini CB, Agnol SMD, Furmann M, et al. Efeito de um programa de fisioterapia aquática no equilíbrio e capacidade funcional de idosos. Sau Pesqu. 2017;10(2):331-8.
4. Demográfico C. Características gerais da população, religião e pessoas com deficiência. Rio de Janeiro: Instituto Brasileiro de Geografia e Estatística. 2010.

5. Organization WH. Global Action Plan Phy Act 20182030: more active people for a healthier world. 2018.

6. Júnior FFG, Brandão AB, de Almeida FJM, de Oliveira JGD. Compreensão de Idosos sobre os Benefícios da Atividade Física. Rev Bra Ciên Saúde. 2016;19(3):1938.

7. Silva RS, Silva Id, Silva RAd, Souza L, Tomasi E. Atividade física e qualidade de vida. Ciên Saúde Col. 2010;15:115-20.

8. Ryan RM, Deci EL. Self-determination theory: Basic psychological needs in motivation, development, and wellness: Guilford Publications; 2017.

9. Deci EL, Ryan RM. Motivation, personality, and development within embedded social contexts: An overview of self-determination theory. Oxfo Hand Hum Moti. 2012:85-107.

10. Fernandes HM, Vasconcelos-Raposo J, Pereira E, Ramalho J, Oliveira S. A influência da actividade física na saúde mental positiva de idosos. Motriz. 2009;5(1):33-50.

11. Bavoso D, Galeote L, Montiel JM, Cecato JF. Motivação e autoestima relacionada à prática de atividade física em adultos e idosos. Revi Bra Psi Espor. 2018;7(2)1-8.

12. Rosário M, Alves S, Cid L, Moutão J. Motivação intrínseca para a prática de atividade física e bem-estar psicológico em indivíduos idosos. Ativ Fís Pop Esp: população idosa, populações com condições clinicas. 2015;2:95-110.

13. Fisher J, Sales A, Carlson L, Steele J. A comparison of the motivational factors between CrossFit participants and other resistance exercise modalities: a pilot study. J Spor Med Phys Fit. 2016;9:1227-34.

14. Marin DP, Polito LFT, Foschini D, Urtado CB, Otton R. Motives, Motivation and Exercise Behavioral Regulations in CrossFit and Resistance Training Participants. Psych. 2018;9:2869-84.

15. Diener E. Subjective well-being: The science of happiness and a proposal for a national index. Ame Psych. $2000 ; 55(1): 34-44$. 
16. Fernandes HM, Vasconcelos-Raposo J, Pereira E, Ramalho J, Oliveira S. A influência da actividade física na saúde mental positiva de idosos. Motri. 2009;5(1):3350 .

17. Mellalieu S, Hanton S. Contemporary advances in sport psychology: A review: Rout; 2015.

18. Smith GL, Banting L, Eime R, O'Sullivan G, van Uffelen JG. The association between social support and physical activity in older adults: a systematic review. Inte J Beh Nutri Phy Acti. 2017;14(1):56-70.

19. de Alencar GP, de Oliveira Marin JL, Medeiros LE. ARTIGO ORIGINAL Perfil de praticantes de treinamento funcional de Campo Grande/MS Functional training practitioners's profile of Campo Grande/MS. Revi Bra Fisi Exe. 2018;17(2):80-5.

20. de Liz CM, Andrade A. Análise qualitativa dos motivos de adesão e desistência da musculação em academias. Revi Bra Ciê Esp. 2016;38(3):267-74.

21. Angleri V, Ugrinowitsch C, Libardi CA. Crescent pyramid and drop-set systems do not promote greater strength gains, muscle hypertrophy, and changes on muscle architecture compared with traditional resistance training in well-trained men. Eur J Appl Physiol. 2017;117(2):359-69.

22. Medicine ACoS. American College of Sports Medicine position stand. Progression models in resistance training for healthy adults. Medi Scie Sports Exerc. 2009;41(3):687.

23. Bossle CB. "Personal trainer \& Cia": noções de marketing na literatura sobre treinamento personalizado. 2009.

24. Thompson WR. Worldwide survey of fitness trends for 2018: the CREP edition. ACSM's Health Fit J. 2017;21(6):10-9.

25. Lawrence RH, Liang J. Structural integration of the Affect Balance Scale and the Life Satisfaction Index A: Race, sex, and age differences. Psychol Aging. 1988;3(4):375-385.

26. Albuquerque AS, Tróccoli BT. Desenvolvimento de uma escala de bem-estar subjetivo. Psic: Teori Pes. 2004;20(2):153-64.

27. Hutz C. Adaptação brasileira da escala de auto-estima de Rosenberg. Manuscrito não-publicado, Curso de Pós-Graduação em Psicologia do Desenvolvimento,
Universidade Federal do Rio Grande do Sul, Porto Alegre, RS. 2000.

28. Markland D, Tobin V. A modification to the behavioural regulation in exercise questionnaire to include an assessment of amotivation. J Spor Exer Psycho. 2004;26(2):191-6.

29. Almeida LM, Pereira HP, Fernandes HM. Efeitos de diferentes tipos de prática desportiva no bem-estar psicológico de jovens estudantes do ensino profissional. Rev Ibero Psi Eje Depo. 2018;13(1):15-21.

30. Westcott WL. Resistance training is medicine: effects of strength training on health. Curr Sport Med Rep. 2012;11(4):209-16.

31. Caudwell KM, Keatley DA. The effect of men's body attitudes and motivation for gym attendance. J Strength Cond Res. 2016;30(9):2550.

32. Silva LXNd. Revisão de literatura acerca do treinamento funcional resistido e seus aspectos motivacionais em alunos de personal training. [monografia] Universidade Federal do Rio Grande do Sul. 2011.63 p.

33. Teixeira PJ, Carraça EV, Markland D, Silva MN, Ryan RM. Exercise, physical activity, and self-determination theory: a systematic review. Int J Behav Nutri Phys Act. 2012;9(1):78-90.

34. Saba F. Aderência: Editora Manole Ltda; 2001.

35. Balbinotti MAA, Barbosa MLL, Balbinotti CAA, Saldanha RP. Motivação à prática regular de atividade física: um estudo exploratório. Est Psi. 2011;16(1):99106.

36. Silva MN, Markland D, Carraça EV, Vieira PN, Coutinho SR, Minderico CS, et al. Exercise autonomous motivation predicts 3-yr weight loss in women. Med Sci Sports Exerc. 2011;43(4):728-37.

Recebido em: 22/03/2019 Aceito em: 25/05/2019 